\title{
A Modified Globally Convergent Self-Scaling BFGS Algorithm for Unconstrained Optimization
}

\author{
Prof. Dr. Abbas Y. AL-Bayati
}

Dr. Basim A. Hassan

Department of Mathematics

College of Computers Sciences and Mathematics

University of Mosul

\author{
\& \\ Sawsan S. Ismael \\ Department of Mathematics / College of Education \\ University of Mosul
}

Received

17 / 10 / 2010
Accepted

06 / 04 / 2011

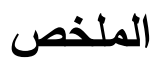

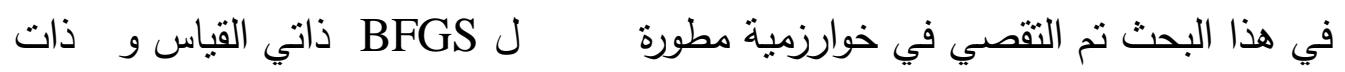

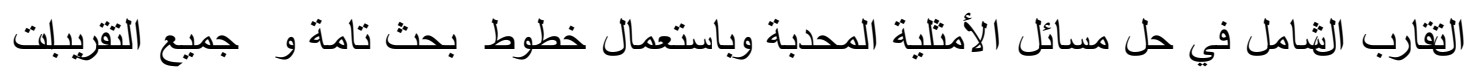

لمعكوس مصفوفة هي موجبة التعريف ـ النتائج العددية أثثتت كفاءة الخوارزمية المقترحة مقارنة بخوارزمية- BFGS - D القياسية.
\end{abstract}

\section{Abstract}

In this paper, a modified globally convergent self-scaling BFGS algorithm for solving convex unconstrained optimization problems was investigated in which it employs exact line search strategy and the inverse Hessian matrix approximations were positive definite. Experimental results indicate that the new proposed algorithm was more efficient than the standard BFGS- algorithm.

\section{Introduction.}

Consider the unconstrained optimization problem

$$
\min \left\{f(x) \mid x \in R^{n}\right\}
$$

where $f$ is a continuously differentiable function of $n$ variables. QuasiNewton (QN) methods for solving (1) often needed to update the 
iterative matrix $B_{k}$. Traditionally, $\left\{B_{k}\right\}$ satisfies the following QN equation:

$$
B_{k+1} v_{k}=y_{k}
$$

where $v_{k}=x_{k+1}-x_{k}, y_{k}=g_{k+1}-g_{k} .[5]$

The search direction is computed by:

$$
d_{k}=-B_{k}^{-1} g_{k}
$$

where $g_{k}$ is the gradient of $f$ evaluated at the current iterate $x_{k}$. One then computes the next iterate by

$$
x_{k+1}=x_{k}+\alpha_{k} d_{k}
$$

where the step size $\alpha_{k}$ satisfies the Wolfe - Powell (WP) conditions

$$
\begin{gathered}
f\left(x_{k}+\alpha_{k} d_{k}\right) \leq f\left(x_{k}\right)+\delta_{1} \alpha_{k} d_{k}^{T} g_{k} \\
g\left(x_{k}+\alpha_{k} d_{k}\right)^{T} d_{k} \geq \delta_{2} d_{k}^{T} g_{k}
\end{gathered}
$$

where $\delta_{1} \prec 1 / 2 \quad$ and $\delta_{1} \prec \delta_{2} \prec 1[2]$.

The famous update $B_{k}$ is the BFGS formula for which $B_{k+1}$ is updated as :

$$
B_{k+1}=B_{k}-\frac{B_{k} v_{k} v_{k}^{T} B_{k}}{v_{k}^{T} B_{k} v_{k}}+\frac{y_{k} y_{k}^{T}}{v_{k}^{T} y_{k}}
$$

In [6] Zhang and $\mathrm{Xu}$ proposed a new $\mathrm{QN}$-condition defined by:

$$
B_{k+1} v_{k}=y_{k}^{*}
$$

By using (8), The modified BFGS-update may be given by:

$$
B_{k+1}=B_{k}-\frac{B_{k} v_{k} v_{k}^{T} B_{k}}{v_{k}^{T} B_{k} v_{k}}+\frac{y_{k}^{*} y_{k}^{* T}}{v_{k}^{T} y_{k}^{*}}
$$

where

$$
y_{k}^{*}=y_{k}+\frac{6\left[f_{k}-f_{k+1}\right]+3\left(g_{k+1}+g_{k}\right)^{T} v_{k}}{v_{k}^{T} y_{k}} y_{k}
$$

Dai [3] proved that updates (9)-(10) may fail for convergence, especially for the non-convex functions. To overcome this drawback, let us consider the self-scaling BFGS update due to Al-Bayati [1].

\subsection{Al-Bayati Self-Scaling BFGS-Method [1].}

The standard BFGS update can be separated into two components, $B^{(1)}$ and $B^{(2)}$ so that:

$$
B_{B F S G}=B^{(1)}+B^{(2)}
$$


where $\quad B^{(1)}=B_{k}-\frac{B_{k} v_{k} v_{k}^{T} B_{k}}{v_{k}^{T} B_{k} v_{k}} \quad, \quad B^{(2)}=\frac{y_{k} y_{k}^{T}}{v_{k}^{T} y_{k}}$

AL-Bayati's [1] modifications for the BFGS formula can then be written as:

$$
B_{A L-\text { Bayati }}=B^{(1)}+\rho_{k} B^{(2)}, \quad \rho_{k}=\frac{v_{k}^{T} B_{k} v_{k}}{y_{k}^{T} v_{k}}
$$

which will satisfy

$$
B_{k+1} v_{k}=\rho y_{k}
$$

This relaxation of the QN-condition is of particular interest in deriving different VM-algorithms for non-quadratic objective functions.

\subsection{A Modified Self-Scaling BFGS-Algorithm.}

In this section we will deal with an algorithm which generates the sequence of $B_{k+1}$ matrices which converge to the Hessian matrix $G$ and satisfies the following modified QN-condition:

$$
B_{k+1} v_{k}=\rho^{*} y_{k}^{*}
$$

According to the above strategy, $B^{(1)}$ and $B^{(2)}$ of Al-Bayati's update can be represented as:

$$
B_{\mathrm{MBFGS}}=B^{(1)}+B^{(2)}
$$

where $\quad B^{(1)}=B_{k}-\frac{B_{k} v_{k} v_{k}^{T} B_{k}}{v_{k}^{T} B_{k} v_{k}} \quad, \quad B^{(2)}=\frac{y_{k}^{*} y_{k}^{* T}}{v_{k}^{T} y_{k}^{*}}$

By using (12), the modified AL-Bayati's BFGS- update may be written as:

$$
B_{k+1}^{n e w}=B_{k}-\frac{B_{k} v_{k} v_{k}^{T} B_{k}}{v_{k}^{T} B_{k} v_{k}}+\rho^{*}{ }_{k} \frac{y_{k}^{*} y_{k}^{* T}}{v_{k}^{T} y_{k}^{*}}
$$

where $\quad \rho^{*}{ }_{k}=\frac{v_{k}^{T} B_{k} v_{k}}{y_{k}^{* T} v_{k}}, y_{k}^{*}$ is a vector defined in (10).

\subsection{Outline of the Modified BFGS-Algorithm.}

The outline of the modified BFGS algorithm is as follows:

Step 0 : Choose an initial point $x_{1} \in R^{n}$ and an initial positive definite matrix $B_{1}=I_{n^{*} n}, \varepsilon=1 * 10^{-4}$, set $k=1$.

Step 1 : If $\left\|g_{k}\right\| \leq \varepsilon$, stop .

Step 2 : Solve $d_{k}=-H_{k} g_{k}$ to obtain a search direction $d_{k}$. 
Step 3 : Find $\alpha_{k}$ by (WP) step-size rules (5) and (6) .

Step 4 : Generate a new iteration point by $x_{k+1}=x_{k}+\alpha_{k} d_{k}$ and calculate the new updating formula (16) with (10).

Step 5 : Set $k=k+1$ and go to Step 1 .

\section{The Global Convergence Property for the Modified BFGS- Algorithm.}

The important property of the line search method is the global convergence property defined by the relation :

$$
\lim _{k \rightarrow \infty} \inf \left\|g_{k}\right\|=0
$$

To prove this property, it is equivalent to prove that the modified BFGS-updating formula (16) generates identical conjugate gradient search directions provided that the function is quadratic and exact line searches are used . Let us consider the following property :

\section{Property (2.1).}

Let $f$ be given by

$$
f(x)=\frac{1}{2} x^{T} G x+b^{T} x
$$

where $G$ is symmetric positive definite. Choose an initial approximation $H_{1}=H$ where $H$ is any symmetric positive definite matrix. Obtain $H_{k+1}$ from $H$ where $d_{k}=-H g_{k}$ is the search direction and assuming exact lime searches then:

$$
H_{k+1} g^{*}=H g^{*}
$$

Proof : see [1].

It is well know that the inverse equivalent BFGS-update formula of the modified updating formula (16) can be written also as :

$$
H_{k+1}=H_{k}-\frac{H_{k} y_{k}^{*} v_{k}^{T}+v_{k} y_{k}^{* T} H_{k}}{y_{k}^{* T} H_{k} y_{k}^{*}}+\frac{v_{k} v_{k}^{T}}{v_{k}^{T} y_{k}^{*}}\left[\rho_{k}^{*}{ }_{k}+\frac{y_{k}^{* T} H_{k} y_{k}^{*}}{v_{k}^{T} y_{k}^{*}}\right]
$$

where $\rho^{*}{ }_{k}=\frac{y_{k}^{* T} H_{k} y_{k}^{*}}{v_{k}^{T} y_{k}^{*}}, y_{k}^{*}$ is a vector defined in (10)

which is the dual of the modified updating formula (16) in sense that $H_{k} \Rightarrow B_{k}, v_{k} \Rightarrow y_{k}$ and $\rho_{k} \Rightarrow \rho_{k}^{-1}$.

The following Lemma (2.1) which was given in [6] page 273, yields: 


\section{Lemma (2.1).}

Suppose that if $\left(\alpha_{k}, x_{k}, y_{k}, d_{k}\right)$ generated by the BFGS update and that $G$ is continuous at $x^{*}$. Then we have

$$
\lim _{k \Rightarrow \infty}\left\|\frac{6\left[f_{k}-f_{k+1}\right]+3\left(g_{k+1}+g_{k}\right)^{T} v_{k}}{v_{k}^{T} y_{k}}\right\|=0
$$

\section{New Theorem (2.1).}

Assume that $f(x)$ be a quadratic function defined in (17b) and that the line searches are exact: let $H$ be any symmetric positive definite matrix for the modified self scaling BFGS-updating formula defined by:

$$
H_{k+1}=H_{k}-\frac{H_{k} y_{k}^{*} v_{k}^{T}+v_{k} y_{k}^{* T} H_{k}}{y_{k}^{* T} H_{k} y_{k}^{*}}+\frac{v_{k} v_{k}^{T}}{v_{k}^{T} y_{k}^{*}}\left[\rho_{k}^{*}{ }_{k}+\frac{y_{k}^{* T} H_{k} y_{k}^{*}}{v_{k}^{T} y_{k}^{*}}\right]
$$

where $\rho^{*}{ }_{k}=\frac{y_{k}^{* T} H_{k} y_{k}^{*}}{v_{k}^{T} y_{k}^{*}}$

Then the search direction

$$
d_{M B F G S}=-H_{k+1} g^{*}
$$

is identical to the Hestenes and Stiefel conjugate gradient direction $d_{H S C G}$ defined by:

$$
d_{H S C G}=-g^{*}+\frac{y^{* T} g^{*}}{y^{* T} d} d \quad \text { for } k \geq 1
$$

\section{Proof :}

$$
H_{k+1}=H_{k}-\frac{H_{k} y_{k}^{*} v_{k}^{T}}{v^{T} y_{k}^{*}}-\frac{v_{k} y_{k}^{* T} H_{k}}{v^{T} y_{k}^{*}}+\frac{v_{k} v_{k}^{T}}{v_{k}^{T} y_{k}^{*}}\left[\rho^{*}{ }_{k}+\frac{y_{k}^{* T} H_{k} y_{k}^{*}}{v_{k}^{T} y_{k}^{*}}\right]
$$

Now

$$
\begin{gathered}
d_{M B F G S}=-H_{k} g_{k}^{*}+\frac{H_{k} y_{k}^{*} v_{k}^{T} g_{k}^{*}}{v_{k}^{T} y_{k}^{*}}+\frac{y_{k}^{* T} H_{k} g_{k}^{*}}{v_{k}^{T} y_{k}^{*}} v_{k}-2 \frac{y_{k}^{* T} H_{k} y_{k}^{*} v_{k}^{T} g_{k}^{*}}{\left(v_{k}^{T} y_{k}^{*}\right)^{2}} v \\
=-H_{k} g_{k}^{*}+\frac{y_{k}^{* T} H_{k} g_{k}^{*}}{v_{k}^{T} y_{k}^{*}} v_{k}
\end{gathered}
$$

using the property $v_{k}^{T} g_{k}^{*}=0$ quoted earlier which holds for exact line searches. The vector $g_{k}^{*}$ can be substituted for $H_{k} g_{k}^{*}$ by using property (2.1). Therefore :

$$
d_{M B F G S}=-g_{k}^{*}+\frac{y_{k}^{* T} g_{k}^{*}}{v_{k \text { new }}^{T} y_{k}^{*}} v_{k}
$$

New by using Lemma (2.1) we have 


$$
d_{M B F G S}=-g_{k}^{*}+\frac{y_{k}^{T} g_{k}^{*}}{v_{k}^{T} y_{k}} v_{k}
$$

We also know that $d_{M B F G S}$ and $d_{H S C G}$ are identical [4] with exact line searches. Hence equation (25) becomes

$$
d_{k+1(M B F G S)}=-g_{k}^{*}+\frac{y_{k}^{T} H_{k} g_{k}^{*}}{d_{k(H S C G)}^{T} y_{k}} d_{k(H S C G)}
$$

This completes the proof of the global convergence property of the modified BFGS-algorithm.

\section{Numerical Results.}

This section was devoted to numerical experiments. Our purpose was to check whether the modified self scaling AL-Bayati's BFGSalgorithm provide improvements on the corresponding standard BFGSalgorithm. The programs were written in Fortran 90. The test functions were commonly used for unconstrained test problems with standard starting points [6] and a summary of the results of these test functions was given in Table (3.1). The same line search was employed in each algorithm, this was the cubic interpolation technique which is satisfy conditions (5) and (6) for convex optimization with $\delta_{1}=0.0001$ and $\delta_{2}=0.1$. The initial inverse approximation was $H_{0}=I$. The stopping criterion was taken to be $\left\|g_{k+1}\right\| \prec 1 * 10^{-4}$. We tabulate for comparison of these algorithms, the number of function evaluations (NOF) and the number of iterations (NOI) .

Table (3.1) gives the comparison between the standard BFGSalgorithm and the modified self-scaling BFGS-algorithm for convex optimization, this table indicates that the modified algorithm saves $11 \%$ NOI and 14\% NOF, overall against the standard BFGS-algorithm, especially for our selected test problems.

\begin{tabular}{|c|c|c|c|c|c|}
\hline \multirow[b]{2}{*}{ Test-functions } & \multirow[b]{2}{*}{$\mathbf{N}$} & \multirow{2}{*}{\multicolumn{2}{|c|}{$\begin{array}{l}\text { Modified algorithm } \\
\text { NOF (NOI) }\end{array}$}} & \multicolumn{2}{|c|}{ BFGS algorithm } \\
\hline & & & & NOF & (NOI) \\
\hline \multirow[t]{5}{*}{ Non-diagonal } & 10 & 99 & $(40)$ & 108 & $(40)$ \\
\hline & 40 & 121 & (53) & 118 & $(50)$ \\
\hline & 100 & 116 & $(51)$ & 135 & $(56)$ \\
\hline & 400 & 119 & $(52)$ & 144 & (59) \\
\hline & 1000 & 127 & $(54)$ & 150 & $(62)$ \\
\hline \multirow[t]{3}{*}{ Wolfe } & 10 & 31 & $(15)$ & 32 & $(15)$ \\
\hline & 40 & 85 & $(42)$ & 87 & (43) \\
\hline & 100 & 113 & (56) & 125 & $(62)$ \\
\hline
\end{tabular}

Table (3.1) 
Prof. Dr. Abbas Y. AL-Bayati \& Dr. Basim A. Hassan \& Sawsan S. Ismael

\begin{tabular}{|c|c|c|c|c|c|}
\hline & 400 & 125 & (62) & 139 & (69) \\
\hline & 1000 & 143 & (71) & 168 & (83) \\
\hline \multirow[t]{5}{*}{ Cubic } & 10 & 61 & (24) & 70 & (26) \\
\hline & 40 & 85 & (36) & 83 & (35) \\
\hline & 100 & 79 & (33) & 98 & (41) \\
\hline & 400 & 80 & (33) & 109 & $(48)$ \\
\hline & 1000 & 101 & $(45)$ & 112 & (48) \\
\hline \multirow[t]{5}{*}{ Powell } & 10 & 82 & (26) & 82 & (23) \\
\hline & 40 & 100 & $(38)$ & 132 & $(46)$ \\
\hline & 100 & 98 & (36) & 140 & (51) \\
\hline & 400 & 113 & (40) & 157 & (54) \\
\hline & 1000 & 103 & (38) & 120 & (39) \\
\hline \multirow[t]{5}{*}{ Cantrell } & 10 & 41 & (10) & 52 & (11) \\
\hline & 40 & 48 & (11) & 52 & (11) \\
\hline & 100 & 54 & (12) & 56 & (12) \\
\hline & 400 & 57 & (13) & 71 & (14) \\
\hline & 1000 & 71 & $(17)$ & 71 & (14) \\
\hline \multirow[t]{5}{*}{ Miele } & 10 & 56 & (19) & 65 & (21) \\
\hline & 40 & 80 & (26) & 92 & $(30)$ \\
\hline & 100 & 76 & (26) & 93 & (30) \\
\hline & 400 & 83 & $(28)$ & 98 & (31) \\
\hline & 1000 & 84 & (28) & 97 & (32) \\
\hline Total & & 2631 & $(1035)$ & 3056 & (1156) \\
\hline
\end{tabular}

The Percentage Performance of the improvements is given by:

\begin{tabular}{|c|c|c|}
\hline Tools & BFGS & Modified algorithm \\
\hline NOI & $100 \%$ & $89.53 \%$ \\
\hline NOF & $100 \%$ & $86.09 \%$ \\
\hline
\end{tabular}

\section{Appendix.}

1.Cubic function :

$$
f(x)=\sum_{i=1}^{n / 2}\left(100\left(x_{2 i}-x_{2 i-1}^{3}\right)^{2}+\left(1-x_{2 i-1}\right)^{2}\right)
$$

Starting point: $(-1.2,1,-1.2,1, \ldots \ldots)^{T}$

2.Non-diagonal function:

$$
f(x)=\sum_{i=1}^{n / 2}\left(100\left(x_{i}-x_{i}^{3}\right)^{2}+\left(1-x_{i}\right)^{2}\right)
$$

Starting point:(-1, )$^{T}$ 
3.Generalized powell function:

$\left.f(x)=\sum_{i=1}^{n / 4}\left(x_{4 i-3}-10 x_{4 i-2}\right)^{2}+5\left(x_{4 i-1}-x_{4 i}\right)^{2}+\left(x_{4 i-1}-2 x_{4 i}\right)^{2}+10\left(x_{4 i-9}-x_{4 i}\right)^{4}+\left(x_{4 i-2}-2 x_{4 i-1}-x_{4 i}\right)^{2}\right)$

Starting point: $(3,1,0,1, \ldots \ldots \ldots \ldots \ldots \ldots . . . . . \ldots)^{T}$

4. Miele function:

$$
f(x)=\sum_{i=1}^{n / 4}\left[\exp \left(x_{4 i-3}\right)-x_{4 i-2}\right]^{2}+100\left(x_{4 i-2}-x_{4 i-1}\right)^{6}+\left[\tan \left(x_{4 i-1}-x_{4 i}\right)\right]^{4}+x_{4 i-3}^{8}+\left(x_{4 i}-1\right)^{2}
$$

Starting point: $(1,2,2,2, \ldots \ldots \ldots \ldots \ldots . . . .)^{T}$

5.Welfe function:

$f(x)=\left(-x_{1}\left(3-x_{1} / 2\right)+2 x_{2}-1\right)^{2}+\sum_{i=1}^{n-1}\left(x_{i-1}-x_{i}\left(3-x_{i}\left(3-x_{i} / 2\right)+2 x_{i+1}-1\right)^{2}+\left(x_{n+1}-x_{n}\left(3 x_{n} / 2-1\right)^{2}\right.\right.$

Starting point: $(-1, \ldots \ldots \ldots \ldots \ldots \ldots \ldots)^{T}$

6.Cantrell function:

$$
f(x)=\sum_{i=1}^{n / 4}\left[\exp \left(x_{4 i-3}\right)-x_{4 i-2}\right]^{4}+100\left(x_{4 i-2}-x_{4 i-1}\right)^{6}+\left[\tan ^{-1}\left(x_{4 i-1}-x_{4 i}\right)\right]^{4}+x_{4 i-3}^{8}
$$

Starting point: $(1,2,2,2, \ldots \ldots \ldots \ldots \ldots . . . .)^{T}$

\section{References.}

1) Al-Bayati, A., (1991), A new family of self-scaling variable metric algorithms for unconstrained optimization, Journal of Educ. and Sci., Iraq, Vol.12, pp.25-54.

2) Al-Bayati, A. and Al-Salih, M., (2008), New self-scaling VMalgorithm for non - convex optimization, to be appear in the 2 nd conference Aleppo- Syria.

3) Dai Y., (2003), Convergence properties of the BFGS algorithm, SIAM J. Optimization, Vol. 13, pp. 693-701.

4) Nazareth, L., (1979), A relationship between BFGS and conjugate gradient algorithm and its implementation for new algorithms, SIAM Journal Numerical Analysis Vol.61, pp.409-436.

5) Zhang, H., Hager, W., (2004), A non-monotone line search technique and its application to unconstrained optimization. SIAM J. Optimization, Vol.14, pp.1043- 1056.

6) Zhang, J., Xu, C., (2001), Properties and numerical performance of quasi - Newton Methods with modified quasi - Newton equations Com. and Applied Mathematics, Vol. 137, pp.269-278. 